Article

\title{
Cyclopalladated ferrocenylimines with ester groups for Heck and Suzuki coupling reactions
}

\author{
Jiong Wang a, Bing Mu ${ }^{\text {b }}$, Zhihua Fua , Li Wanga , Tiesheng $\mathrm{Li}^{\mathrm{a}}{ }^{\text {,*}}$, Yangjie Wu ${ }^{\text {a,\# }}$ \\ a The College of Chemistry and Molecular Engineering, The Key Lab of Chemical Biology and Organic Chemistry of Henan Province, The Key Lab of \\ Advanced Nano-information Materials of Zhengzhou, Zhengzhou University, Zhengzhou 450001, Henan, China \\ b Department of Chemistry, Zhengzhou Normal University, Zhengzhou 450044, Henan, China
}

\section{A R T I C L E I N F O}

Article history:

Received 10 November 2013

Accepted 22 January 2014

Published 20 July 2014

Keywords:

Heck coupling reaction

Suzuki coupling reaction

Cyclopalladated ferrocenylimine

\section{A B S T R A C T}

Cyclopalladated ferrocenylimine dimers and cyclopalladated ferrocenylimine monomers were designed and prepared. They were characterized using ${ }^{1} \mathrm{H}$ NMR, ${ }^{13} \mathrm{C}$ NMR, and IR spectroscopies, and MS. The catalytic properties of these compounds in Heck and Suzuki coupling reactions in water or organic solvents were systematically investigated. The results showed that these catalysts could effectively catalyze Heck and Suzuki coupling reactions of aryl or heteroaryl halides in water or organic solvents.

(C) 2014, Dalian Institute of Chemical Physics, Chinese Academy of Sciences. Published by Elsevier B.V. All rights reserved.

\section{Introduction}

The palladium-catalyzed Suzuki and Heck coupling reactions of aryl halides are important and versatile methods for the generation of $\mathrm{C}-\mathrm{C}$ bonds [1-7] and have been used extensively in the synthesis of natural products, pharmaceuticals, herbicides, and advanced materials [8,9]. The Suzuki coupling reaction is useful because of its tractability, high stability, mild reaction conditions, and broad functional group tolerance, as well as the low toxicity of boron compounds [10-14]. Generally, palladium-catalyzed Heck and Suzuki reactions are performed in polar organic solvents under oxygen-free conditions because of the solubility of the substrate and instability of most catalysts. However, from economic and environmental standpoints, it is desirable to use water as a solvent instead of hazardous and expensive organic solvents [15]. It is therefore worthwhile developing new highly active catalysts that are not sensitive to air and moisture, as well as easy to recover and recycle [16-19]. Some significant advances have been made in meeting these requirements, including the use of phase-transfer catalysts [20-22], water-soluble phosphine ligands [23,24], water as a cosolvent [25,26], and amphiphilic polymers for catalyst immobilization [27]. More recently, microwave heating [28] and ultrasonic irradiation [29] have been used as tools in synthetic chemistry to facilitate $\mathrm{C}-\mathrm{C}$ coupling in water. However, many of these procedures use expensive and complex ligands, supports, or external assistance such as microwave heating or ultrasonic irradiation, and, with a few exceptions, most reactions involve hazardous organic cosolvents. To the best of our knowledge, only a few studies have shown that Suzuki and Heck coupling reactions could be carried out efficiently using ligand-free palladium catalysts under conventional heating in neat water. For instance, the protocol for a Suzuki coupling reaction using $2.5 \mathrm{~mol} \%$ of $\mathrm{Na}_{2} \mathrm{PdCl}_{4}$, with the addition of so-

\footnotetext{
* Corresponding author. Tel/Fax +86-371-67766667; E-mail: Its34@zzu.edu.cn

\# Corresponding author. Tel/Fax +86-371-67766667; E-mail: wyj@zzu.edu.cn

This work was supported by the National Natural Science Foundation of China (20973157, J1210060) and the Research and Development Foundation of Zhengzhou University (2013xjxm020).

DOI: 10.1016/S1872-2067(14)60045-0 | http://www.sciencedirect.com/science/journal/18722067 | Chin. J. Catal., Vol. 35, No. 7, July 2014
} 
dium dodecyl sulfate as a surfactant, in pure water under aerobic conditions at $100{ }^{\circ} \mathrm{C}$ was reported by Ranu et al. [20]. Marziale et al. [30] developed an efficient method for Suzuki coupling reactions catalyzed by a palladacyclic catalyst precursor produced by ortho palladation of ([1,1'-biphenyl $]$-2-yloxy) diisopropylphosphine, enabling the reaction to be performed in neat water under air and at ambient temperature with $\mathrm{Pd}$ loadings of $0.04 \mathrm{~mol} \%$. Basu et al. [21] found that a combination of $\mathrm{Pd}(\mathrm{OAc})_{2}$ and tetra- $n$-butylammonium bromide in water at ambient temperature showed high catalytic activity in the reactions of aryl bromides or iodides with aryl trihydroxyborate salts of sodium at catalyst loading of $0.5 \mathrm{~mol} \%$. The key to the coupling reaction was the use of aryl trihydroxyborate salts of sodium to increase the substrate solubility in pure water. The protocol for Suzuki and Heck reactions catalyzed by 0.1 mol\% palladium supported on a magnetic microgel in water at $90{ }^{\circ} \mathrm{C}$ was reported by $\mathrm{Yu}$ et al. [31]; however, the attractiveness of their method is limited because it requires an argon atmosphere. In addition, Roglans et al. [32] demonstrated that $\mathrm{PdCl}_{2}\left(\mathrm{CH}_{3} \mathrm{CN}\right)_{2}$ was an efficient catalyst for Suzuki and Heck coupling reactions in water at room temperature or $40{ }^{\circ} \mathrm{C}$; however, arenediazonium salts were used as substrates, and the amount of palladium catalyst used was high, 5-10 mol\%. From the academic and industrial viewpoints, the design of catalytic systems using simple ligand-free palladium catalysts without other external assistance in neat water is therefore still a promising route for establishing greener $\mathrm{C}-\mathrm{C}$ coupling reactions [22].

In our previous studies, we synthesized a series of cyclopalladated ferrocenylimines, which showed satisfactory reactivities in Heck, Suzuki, Sonogashira, and Kumada reactions [33-41]. However, there has recently been considerable interest in the development of new and simple catalysts that can effectively catalyze $\mathrm{C}-\mathrm{C}$ coupling reactions in water or aqueous media because such catalysts have the potential to be used in industrial systems. In this paper, the preparation and catalytic properties of cyclopalladated ferrocenylimines with ester groups are reported. The results show that these compounds effectively catalyze Heck and Suzuki coupling reactions with catalyst loadings of $0.1 \mathrm{~mol} \%$ under ligand-free and ambient conditions in water or organic solvents.

\section{Experimental}

\subsection{General}

All chemicals were purchased from commercial companies. $\mathrm{N}, \mathrm{N}$-Dimethylformamide (DMF), toluene, and methanol were dried with $\mathrm{MgSO}_{4}$, filtered, distilled, and stored over $4 \AA$ A molecular sieves. Melting points were measured using a WC-1 microscopic apparatus and were uncorrected. IR spectra were recorded with a Bruker VECTOR22 spectrometer using $\mathrm{KBr}$ pellets. Mass spectra were recorded using an LC-MSD-TrapXCT instrument. ${ }^{1} \mathrm{H}$ and ${ }^{13} \mathrm{C}$ NMR spectra were recorded in $\mathrm{CDCl}_{3}$ using a Bruker DPX-400 spectrometer with tetramethylsilane (TMS) as the internal standard. The GC yields were determined using $n$-dodecane internal standards. GC analyses were carried out using a Shimadzu GC-2010 Plus instrument. All the Suzuki and Heck coupling reactions were performed without the protection of an inert gas.

\subsection{Preparation of compounds}

Cyclopalladated ferrocenylimines were prepared as shown in Scheme 1.

\subsubsection{Preparation of ferrocenylimines}

A mixture of acetylferrocene (5.0 mmol), $p$-aminoethylbenzoate $(5.0 \mathrm{mmol})$ or $m$-aminoethylbenzoate $(5.0 \mathrm{mmol})$, molecular sieve ( $4 \AA, 4.0 \mathrm{~g}$ ), and toluene ( $80 \mathrm{~mL}$ ) was refluxed for 24 $\mathrm{h}$ at $120{ }^{\circ} \mathrm{C}$ under a nitrogen atmosphere. The volume of the solution was reduced to ca. $10 \mathrm{~mL}$. The products were separated and purified on a chromatography column with petroleum ether and ethyl acetate (2:1) as the eluent.

$\left[\left(\eta^{5}-\mathrm{C}_{5} \mathrm{H}_{5}\right) \mathrm{Fe}\left(\eta^{5}-\mathrm{C}_{5} \mathrm{H}_{4} \mathrm{CMe}=\mathrm{NC}_{6} \mathrm{H}_{4}-p\right.\right.$-COOEt $\left.)\right] . \quad$ Yield, 80\%; deep-red solid; m.p. $122-124{ }^{\circ} \mathrm{C}$; IR ( $\mathrm{KBr}$ pellet): 2922, 2846, $1704,1627,1594,1100,816,714 \mathrm{~cm}^{-1} ;{ }^{1} \mathrm{H}$ NMR $(400 \mathrm{MHz}$, $\left.\mathrm{CDCl}_{3}\right): \delta 8.04-8.02\left(2 \mathrm{H}, \mathrm{m}, \mathrm{C}_{6} \mathrm{H}_{4}\right), 6.80\left(2 \mathrm{H}, \mathrm{d}, J=8.4 \mathrm{~Hz}, \mathrm{C}_{6} \mathrm{H}_{4}\right)$, $4.79\left(2 \mathrm{H}, \mathrm{t}, J=1.8 \mathrm{~Hz}, \mathrm{C}_{5} \mathrm{H}_{4}\right), 4.46\left(2 \mathrm{H}, \mathrm{t}, J=1.8 \mathrm{~Hz}, \mathrm{C}_{5} \mathrm{H}_{4}\right)$, 4.40-4.35 (2H, m, $\left.\mathrm{CH}_{2} \mathrm{CH}_{3}\right), 4.23\left(5 \mathrm{H}, \mathrm{s}, \mathrm{C}_{5} \mathrm{H}_{5}\right), 2.08(3 \mathrm{H}, \mathrm{s}$,<smiles></smiles>

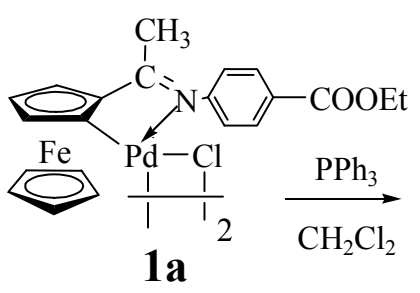

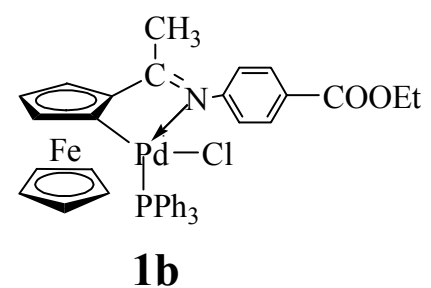

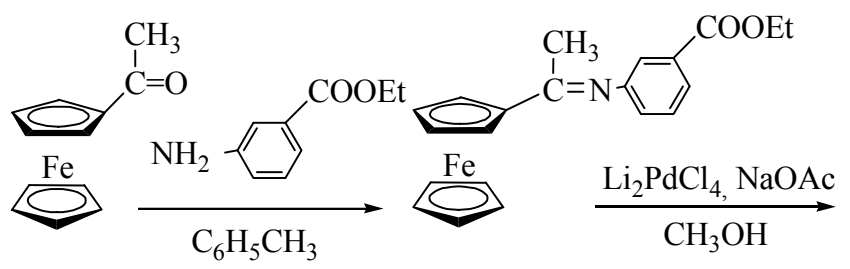
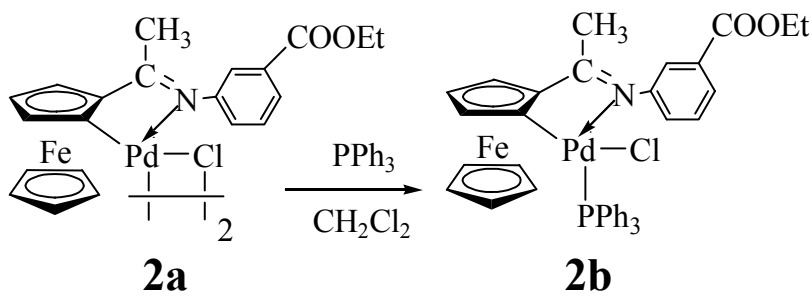

Scheme 1. Syntheses of cyclopalladated ferrocenylimines. 
$\left.\mathrm{CH}_{3}-\mathrm{C}=\mathrm{N}-\right), 1.40\left(3 \mathrm{H}, \mathrm{t}, J=5.2 \mathrm{~Hz}, \mathrm{CH}_{2} \mathrm{CH}_{3}\right) ;{ }^{13} \mathrm{C} \mathrm{NMR}(100 \mathrm{MHz}$, $\left.\mathrm{CDCl}_{3}\right): \delta 168.1,166.6,156.3,130.7,125.1,119.4,83.0,71.0$, 69.9, 69.6, 68.4, 60.7, 58.5, 30.1, 18.3, 14.4; MS: Calcd for $\mathrm{C}_{21} \mathrm{H}_{21} \mathrm{FeNO}_{2}[M+1]+376.1$, found 376.7 .

$\left[\left(\eta^{5}-\mathrm{C}_{5} \mathrm{H}_{5}\right) \mathrm{Fe}\left(\eta^{5}-\mathrm{C}_{5} \mathrm{H}_{4} \mathrm{CMe}=\mathrm{NC}_{6} \mathrm{H}_{4}-m\right.\right.$-COOEt $\left.)\right]$. Yield, 85\%; red oil; IR (KBr pellet): 2922, 2846, 1704, 1627, 1594, 1461, 1365, 880, 780, $700 \mathrm{~cm}^{-1}$; ${ }^{1} \mathrm{H}$ NMR (400 MHz, $\mathrm{CDCl}_{3}$ ): $\delta 7.74$ $\left(1 \mathrm{H}, \mathrm{d}, J=7.1 \mathrm{~Hz}, \mathrm{C}_{6} \mathrm{H}_{4}\right), 7.40\left(1 \mathrm{H}, \mathrm{s}, \mathrm{C}_{6} \mathrm{H}_{4}\right), 7.30(1 \mathrm{H}, \mathrm{t}, J=7.4 \mathrm{~Hz}$, $\left.\mathrm{C}_{6} \mathrm{H}_{4}\right), 6.90\left(1 \mathrm{H}, \mathrm{d}, J=7.7 \mathrm{~Hz}, \mathrm{C}_{6} \mathrm{H}_{4}\right), 4.79\left(2 \mathrm{H}, \mathrm{s}, \mathrm{C}_{5} \mathrm{H}_{4}\right), 4.45(2 \mathrm{H}$, m, $\left.\mathrm{C}_{5} \mathrm{H}_{4}\right), 4.30-4.25\left(2 \mathrm{H}, \mathrm{m}, \mathrm{CH}_{2} \mathrm{CH}_{3}\right), 4.20\left(5 \mathrm{H}, \mathrm{s}, \mathrm{C}_{5} \mathrm{H}_{5}\right), 2.22$ $\left(3 \mathrm{H}, \mathrm{s}, \mathrm{CH}_{3}-\mathrm{C}=\mathrm{N}-\right), 1.29\left(3 \mathrm{H}, \mathrm{t}, J=7.1 \mathrm{~Hz}, \mathrm{CH}_{2} \mathrm{CH}_{3}\right) ;{ }^{13} \mathrm{CNMR}$ $\left(100 \mathrm{MHz} \mathrm{CDCl}_{3}\right): \delta 166.0,164.6,160.4,148.3,131.0,129.5$, 120.6, 114.3, 80.8, 70.2, 68.3, 62.0, 60.9, 20.5, 14.1; MS: Calcd for $\mathrm{C}_{21} \mathrm{H}_{21} \mathrm{FeNO}_{2}[M+1]+376.1$, found 376.7 .

\subsubsection{Preparation of cyclopalladated ferrocenylimines}

Cyclopalladated dimers (1a and $\mathbf{2 a}$ ) were prepared as follows. A mixture of $\mathrm{Li}_{2} \mathrm{PdCl}_{4}(1.0 \mathrm{mmol})$, sodium acetate (1.0 $\mathrm{mmol})$, and ferrocenylimine $(1.1 \mathrm{mmol})$ in methanol $(10 \mathrm{~mL})$ was stirred for $24 \mathrm{~h}$ at room temperature. The mixture was filtered and the obtained solid was washed with ethanol. The products were separated and purified by chromatography column, using dichloromethane and ethanol (2:1) as the eluent.

$\left[\mathrm{PdCl}\left\{\left[\left(\eta^{5}-\mathrm{C}_{5} \mathrm{H}_{5}\right)\right] \mathrm{Fe}\left[\left(\eta^{5}-\mathrm{C}_{5} \mathrm{H}_{3}\right) \mathrm{CMe}=\mathrm{NC}_{6} \mathrm{H}_{4}-p-\mathrm{COOEt}\right]\right\}\right]_{2} \quad \mathbf{1 a}$ Yield, 90\%; deep-red solid; m.p. $>218{ }^{\circ} \mathrm{C}$ (dec); IR (KBr pellet): 2925, 1274, 1168, 1104, 1017, $816 \mathrm{~cm}^{-1}$; ${ }^{1} \mathrm{H}$ NMR $(400 \mathrm{MHz}$, $\left.\mathrm{CDCl}_{3}\right): \delta 8.09\left(2 \mathrm{H}, \mathrm{d}, J=7.4 \mathrm{~Hz}, \mathrm{C}_{6} \mathrm{H}_{4}\right), 7.14(2 \mathrm{H}, \mathrm{d}, J=7.2 \mathrm{~Hz}$, $\left.\mathrm{C}_{6} \mathrm{H}_{4}\right), 4.66\left(1 \mathrm{H}, \mathrm{s}, \mathrm{C}_{5} \mathrm{H}_{3}\right), 4.41\left(5 \mathrm{H}, \mathrm{s}, \mathrm{C}_{5} \mathrm{H}_{5}\right), 4.38\left(1 \mathrm{H}, \mathrm{s}, \mathrm{C}_{5} \mathrm{H}_{3}\right)$, $4.33\left(1 \mathrm{H}, \mathrm{s}, \mathrm{C}_{5} \mathrm{H}_{3}\right)$ 4.27-4.21 (2H, m, $\left.\mathrm{CH}_{2} \mathrm{CH}_{3}\right), 1.99(3 \mathrm{H}, \mathrm{s}$, $\left.\mathrm{CH}_{3}-\mathrm{C}=\mathrm{N}-\right), 1.43\left(3 \mathrm{H}, \mathrm{t}, J=6.8 \mathrm{~Hz}, \mathrm{CH}_{3} \mathrm{CH}_{2}\right) ;{ }^{13} \mathrm{C} \mathrm{NMR}(100 \mathrm{MHz}$, $\left.\mathrm{CDCl}_{3}\right): \delta 179.8,166.5,165.1,153.4,131.4,128.9,122.4,101.2$ 91.5, 73.8, 71.3, 66.4, 65.9, 61.1, 53.2, 52.4, 21.6, 14.9; MS: Calcd for $\mathrm{C}_{42} \mathrm{H}_{40} \mathrm{Cl}_{2} \mathrm{Fe}_{2} \mathrm{~N}_{2} \mathrm{O}_{4} \mathrm{Pd}_{2}[M-\mathrm{Cl}]+994.9$, found 995.1 .

$\left[\mathrm{PdCl}\left\{\left[\left(\eta^{5}-\mathrm{C}_{5} \mathrm{H}_{5}\right)\right] \mathrm{Fe}\left[\left(\eta^{5}-\mathrm{C}_{5} \mathrm{H}_{3}\right) \mathrm{CMe}=\mathrm{NC}_{6} \mathrm{H}_{4}-m-\mathrm{COOEt}\right]\right\}\right]_{2}$ 2a. Yield, 80\%; deep-red solid; m.p. > $260{ }^{\circ} \mathrm{C}$ (dec); IR (KBr pellet): 2925, 1274, 1168, 1104, 1017, 880, 780, $700 \mathrm{~cm}^{-1}$; ${ }^{1} \mathrm{H}$ NMR $\left(400 \mathrm{MHz}, \mathrm{CDCl}_{3}\right): \delta 7.76\left(1 \mathrm{H}, \mathrm{d}, J=7.8 \mathrm{~Hz}, \mathrm{C}_{6} \mathrm{H}_{4}\right), 7.45(1 \mathrm{H}, \mathrm{s}$, $\left.\mathrm{C}_{6} \mathrm{H}_{4}\right), 7.39\left(1 \mathrm{H}, \mathrm{t}, J=7.8 \mathrm{~Hz}, \mathrm{C}_{6} \mathrm{H}_{4}\right), 6.95\left(1 \mathrm{H}, \mathrm{d}, J=7.8 \mathrm{~Hz}, \mathrm{C}_{6} \mathrm{H}_{4}\right)$, 4.81-4.78 (2H, m, $\left.\mathrm{C}_{5} \mathrm{H}_{5}\right), 4.46-4.41\left(2 \mathrm{H}, \mathrm{m}, \mathrm{C}_{5} \mathrm{H}_{3}\right), 4.37$ (2H, q, J $\left.=7.1 \mathrm{~Hz}, \mathrm{CH}_{2} \mathrm{CH}_{3}\right), 4.23\left(5 \mathrm{H}, \mathrm{s}, \mathrm{C}_{5} \mathrm{H}_{3}\right), 2.08\left(3 \mathrm{H}, \mathrm{s}, \mathrm{CH}_{3}-\mathrm{C}=\mathrm{N}-\right)$, $1.38\left(3 \mathrm{H}, \mathrm{t}, J=7.1 \mathrm{~Hz}, \mathrm{CH}_{2} \mathrm{CH}_{3}\right) ;{ }^{13} \mathrm{C}$ NMR $\left(100 \mathrm{MHz}, \mathrm{CDCl}_{3}\right): \delta$ 179.8, 166.5, 165.1, 153.4, 131.4, 128.9, 122.4, 101.2, 91.5, 73.8, 71.3, 66.4, 61.1, 53.2, 52.4, 21.6, 14.9; MS: Calcd for $\mathrm{C}_{42} \mathrm{H}_{40} \mathrm{Cl}_{2} \mathrm{Fe}_{2} \mathrm{~N}_{2} \mathrm{O}_{4} \mathrm{Pd}_{2}[M-\mathrm{Cl}]+994.9$, found 995.1.

Cyclopalladated ferrocenylimine monomers (1b and $\mathbf{2 b}$ ) were prepared as follows. A mixture of $1 \mathbf{a}$ or $\mathbf{2 a}(0.5 \mathrm{mmol})$ and triphenylphsophine $(1.5 \mathrm{mmol})$ in dichloromethane $(10 \mathrm{~mL})$ was stirred for $1 \mathrm{~h}$. The volume of the solution was reduced to ca. $3 \mathrm{~mL}$. The products were purified on a chromatography column with dichloromethane as the eluent.

$\left[\mathrm{PdCl}\left\{\left[\left(\eta^{5}-\mathrm{C}_{5} \mathrm{H}_{5}\right)\right] \mathrm{Fe}\left[\left(\eta^{5}-\mathrm{C}_{5} \mathrm{H}_{3}\right) \mathrm{CMe}=\mathrm{NC}_{6} \mathrm{H}_{4}-p\right.\right.\right.$-COOEt $\left.\left.]\right\}\left(\mathrm{PPh}_{3}\right)\right]$ 1b. Yield, 91\%; deep-red solid; m.p. > $180{ }^{\circ} \mathrm{C}(\mathrm{dec})$; IR ( $\mathrm{KBr}$ pellet): 2925, 1274, 1168, 1104, 1017, 810, 760, $684 \mathrm{~cm}^{-1} ;{ }^{1} \mathrm{H}$ NMR (400 MHz, $\left.\mathrm{CDCl}_{3}\right): \delta 8.06\left(2 \mathrm{H}, \mathrm{d}, J=8.4 \mathrm{~Hz}, \mathrm{C}_{6} \mathrm{H}_{4}\right)$, 7.81-7.71 (6H, m, $\left.\mathrm{PPh}_{3}\right), 7.43-7.35$ (9H, m, $\left.\mathrm{PPh}_{3}\right), 7.08(2 \mathrm{H}, \mathrm{d}, J$ $\left.=8.1 \mathrm{~Hz}, \mathrm{C}_{6} \mathrm{H}_{4}\right), 4.48\left(1 \mathrm{H}, \mathrm{d}, J=2.2 \mathrm{~Hz}, \mathrm{C}_{5} \mathrm{H}_{3}\right), 4.35(2 \mathrm{H}, \mathrm{m}$, $\left.\mathrm{CH}_{2} \mathrm{CH}_{3}\right), 4.16\left(1 \mathrm{H}, \mathrm{t}, J=2.2 \mathrm{~Hz}, \mathrm{C}_{5} \mathrm{H}_{3}\right), 3.94\left(5 \mathrm{H}, \mathrm{s}, \mathrm{C}_{5} \mathrm{H}_{5}\right), 3.43$ $\left(1 \mathrm{H}, \mathrm{d}, J=2.2 \mathrm{~Hz}, \mathrm{C}_{5} \mathrm{H}_{3}\right), 2.08\left(3 \mathrm{H}, \mathrm{s}, \mathrm{CH}_{3}-\mathrm{C}=\mathrm{N}-\right), 1.36(3 \mathrm{H}, \mathrm{t}, J=$ $7.1 \mathrm{~Hz}, \mathrm{CH}_{3} \mathrm{CH}_{2}$ ); ${ }^{13} \mathrm{CNMR}\left(100 \mathrm{MHz}, \mathrm{CDCl}_{3}\right): \delta 179.8,166.5$, $165.1,153.4,135.0,132.4,131.4,130.3,128.9,127.9,122.4$, 101.2, 91.5, 73.8, 71.3, 66.4, 61.1, 53.2, 52.4, 21.6, 14.9; MS: Calcd for $\mathrm{C}_{39} \mathrm{H}_{35} \mathrm{ClFeNO}_{2} \mathrm{PPd}[\mathrm{M}-\mathrm{Cl}]+742.1$, found 742.1.

$\left[\mathrm{PdCl}\left\{\left[\left(\eta^{5}-\mathrm{C}_{5} \mathrm{H}_{5}\right)\right] \mathrm{Fe}\left[\left(\eta^{5}-\mathrm{C}_{5} \mathrm{H}_{3}\right) \mathrm{CMe}=\mathrm{NC}_{6} \mathrm{H}_{4}-m-\mathrm{COOEt}\right]\right\}\left(\mathrm{PPh}_{3}\right)\right]$ 2b. Yield, 79\%; deep-red solid; m.p. $138-140{ }^{\circ} \mathrm{C}$; IR (KBr pellet): $2925,1274,1168,1104,1017,880,780,700 \mathrm{~cm}^{-1}$; ${ }^{1} \mathrm{H} \mathrm{NMR}$ (400 MHz, $\left.\mathrm{CDCl}_{3}\right): \delta 7.87\left(1 \mathrm{H}, \mathrm{d}, J=7.8 \mathrm{~Hz}, \mathrm{C}_{6} \mathrm{H}_{4}\right), 7.80-7.71$ $\left(6 \mathrm{H}, \mathrm{m}, \mathrm{PPh}_{3}\right), 7.65\left(1 \mathrm{H}, \mathrm{s}, \mathrm{C}_{6} \mathrm{H}_{4}\right), 7.48-7.37\left(9 \mathrm{H}, \mathrm{m}, \mathrm{PPh}_{3}\right)$, 7.37-7.34 (1H, m, $\left.\mathrm{C}_{6} \mathrm{H}_{4}\right), 7.26\left(1 \mathrm{H}, \mathrm{s}, \mathrm{C}_{6} \mathrm{H}_{4}\right), 5.29\left(1 \mathrm{H}, \mathrm{s}, \mathrm{C}_{5} \mathrm{H}_{3}\right)$, $4.48\left(1 \mathrm{H}, \mathrm{d}, J=2.3 \mathrm{~Hz}, \mathrm{C}_{5} \mathrm{H}_{3}\right), 4.35\left(2 \mathrm{H}, \mathrm{q}, J=7.1 \mathrm{~Hz}, \mathrm{CH}_{2} \mathrm{CH}_{3}\right)$, $4.16\left(1 \mathrm{H}, \mathrm{t}, J=2.0 \mathrm{~Hz}, \mathrm{C}_{5} \mathrm{H}_{3}\right), 3.94\left(5 \mathrm{H}, \mathrm{s}, \mathrm{C}_{5} \mathrm{H}_{5}\right), 2.09(3 \mathrm{H}, \mathrm{s}$, $\left.\mathrm{CH}_{3}-\mathrm{C}=\mathrm{N}-\right), 1.38\left(3 \mathrm{H}, \mathrm{t}, \mathrm{J}=7.1 \mathrm{~Hz}, \mathrm{CH}_{2} \mathrm{CH}_{3}\right) ;{ }^{13} \mathrm{C} \mathrm{NMR}(100 \mathrm{MHz}$, $\left.\mathrm{CDCl}_{3}\right): \delta 179.8,166.0,148.3,132.4,131.0,130.3,128.0,127.9$, 120.6, 119.9, 114.3, 101.2, 91.5, 73.8, 71.3, 66.4, 60.9, 53.2, 52.4, 14.1; MS: Calcd for $\mathrm{C}_{39} \mathrm{H}_{35} \mathrm{ClFeNO}_{2} \mathrm{PPd}[M-\mathrm{Cl}]+742.1$, found 742.1.

\subsection{General procedure for Suzuki coupling reactions of aryl bromides with phenylboronic acid}

A mixture of aryl bromide $(0.5 \mathrm{mmol})$, phenylboronic acid $(0.6 \mathrm{mmol})$, base $(1 \mathrm{mmol})$, catalyst $1 \mathrm{a}(0.1 \mathrm{~mol} \%)$, and solvent ( $2 \mathrm{~mL}$ ) was stirred at a specified temperature under air. The reaction mixture was stirred for $2 \mathrm{~h}$ and then diluted with water and ethyl acetate. The organic layer was separated, and the aqueous layer was extracted three times with ethyl acetate. The combined organic phase was dried with $\mathrm{MgSO}_{4}$ and filtered; the solvent was removed using a rotary evaporator. The crude product was transferred to a $10 \mathrm{~mL}$ volumetric flask to fix its quantity in ethyl acetate. Yields of the desired cross-coupling products were determined by GC using $n$-dodecane $(10 \mathrm{~mL}, 0.5$ $\mathrm{mmol}$ ) as an internal standard. The products were isolated using thin-layer chromatography. The ${ }^{1} \mathrm{H}$ NMR spectroscopy and melting point data of the products were identical to those of commercially available authentic samples.

\subsection{General procedure for Heck reactions of aryl halides with styrene}

An aryl halide $(0.5 \mathrm{mmol})$ and styrene $(2 \mathrm{mmol})$ were combined with a base $(0.5 \mathrm{mmol}), n-\mathrm{Bu} 4 \mathrm{NBr}(0.5 \mathrm{mmol})$, and catalyst $1 \mathrm{a}(0.1 \mathrm{~mol} \%)$ in a small round-bottomed flask. The reaction mixture was stirred at a specified temperature under air for $6 \mathrm{~h}$ and then diluted with water and ethyl acetate. The organic layer was separated, and the aqueous layer was extracted three times with ethyl acetate. The combined organic phase was dried with $\mathrm{MgSO}_{4}$ and filtered, and the solvent was removed using a rotary evaporator. The crude product was transferred to a $10 \mathrm{~mL}$ volumetric flask to fix its quantity in ethyl acetate. Yields of the desired cross-coupling products were determined by GC using $n$-dodecane $(10 \mathrm{~mL}, 0.5 \mathrm{mmol})$ as an internal standard. The products were isolated using thin-layer chromatography. The ${ }^{1} \mathrm{H}$ NMR spectroscopy and melting point data of the products were identical to those of commercially available authentic samples. 


\section{Results and discussion}

\subsection{Heck coupling reactions in organic solvents}

The coupling of 4-bromobenzene with styrene catalyzed by 1a was selected as a model reaction. As shown in Table 1, $\mathrm{NaOAc}$ as the base and DMF as the solvent afforded the highest yield (Table 1, entry 8). The relative activities of palladacycles $\mathbf{1 a}, \mathbf{1 b}, \mathbf{2 a}$, and $\mathbf{2 b}$ in the coupling reaction of 4-bromobenzene with styrene in the presence of NaOAc as the base in DMF were examined. The results showed that the catalytic activities of these cyclopalladated complexes were similar, but catalyst 1a was slightly more active under the same reaction conditions (entries 8-11).

The couplings of a number of other aryl bromides were conducted in the presence of $0.1 \mathrm{~mol} \% \mathrm{1a}$, using the optimized conditions described above; the results are shown in Table 2. The reactions of aryl bromides containing electron-withdrawing groups such as $-\mathrm{CN},-\mathrm{CHO},-\mathrm{NO}_{2}$, and $-\mathrm{CF}_{3}$ gave the coupling products in modest to high yields (entries 1and 8-17). Aryl bromides with electron-rich groups such as $-\mathrm{OMe},-\mathrm{NH}_{2}$, and $-\mathrm{CH}_{3}$ were poor coupling partners or almost inactive under the same reaction conditions (entries 2-7). When heterocyclic compounds such as 1-bromonaphthalene, 3-bromothiophene,
Table 1

Investigation of Heck cross-coupling reaction conditions.

\begin{tabular}{|c|c|c|c|c|c|c|}
\hline Entry & Base & Solvent & $\begin{array}{c}T \\
\left({ }^{\circ} \mathrm{C}\right) \\
\end{array}$ & $\begin{array}{c}\text { Yield a }^{a} \\
(\%)\end{array}$ & TON & $\begin{array}{l}\text { TOF } \\
\left(\mathrm{h}^{-1}\right) \\
\end{array}$ \\
\hline 1 & $\mathrm{Na}_{2} \mathrm{CO}_{3}$ & DMF & 130 & 91 & & \\
\hline 2 & $\mathrm{~K}_{3} \mathrm{PO}_{4}$ & DMF & 130 & 66 & & \\
\hline 3 & $\mathrm{KOH}$ & DMF & 130 & 61 & & \\
\hline 4 & $\mathrm{~K}_{3} \mathrm{PO}_{4} \cdot 3 \mathrm{H}_{2} \mathrm{O}$ & DMF & 130 & 48 & & \\
\hline 5 & $\mathrm{Et}_{3} \mathrm{~N}$ & DMF & 130 & 35 & & \\
\hline 6 & $\mathrm{~K}_{2} \mathrm{CO}_{3}$ & DMF & 130 & 30 & & \\
\hline 7 & $\mathrm{NaHCO}_{3}$ & DMF & 130 & 87 & & \\
\hline 8 & $\mathrm{NaOAc}$ & DMF & 130 & $>99$ & 990 & 165 \\
\hline $9^{b}$ & $\mathrm{NaOAc}$ & DMF & 130 & 95 & 950 & 158 \\
\hline $10^{c}$ & $\mathrm{NaOAc}$ & DMF & 130 & 98 & 980 & 163 \\
\hline $11^{\mathrm{d}}$ & $\mathrm{NaOAc}$ & DMF & 130 & 94 & 940 & 157 \\
\hline 12 & $\mathrm{NaOAc}$ & dioxane & 110 & 74 & & \\
\hline 13 & $\mathrm{NaOAc}$ & $\mathrm{CH}_{3} \mathrm{OH}: \mathrm{H}_{2} \mathrm{O}=1: 1$ & 90 & 94 & & \\
\hline 14 & $\mathrm{NaOAc}$ & DMSO & 140 & 16 & & \\
\hline 15 & $\mathrm{NaOAc}$ & toluene & 110 & 6 & & \\
\hline 16 & $\mathrm{NaOAc}$ & methanol & 70 & 72 & & \\
\hline 17 & $\mathrm{NaOAc}$ & THF & 70 & 65 & & \\
\hline
\end{tabular}

Reaction conditions: $\mathrm{PhBr}(0.5 \mathrm{mmol})$, styrene $(2.0 \mathrm{mmol})$, catalyst 1a (0.1 mol\%), base (0.5 mmol), $n-\mathrm{Bu}_{4} \mathrm{NBr}(0.5 \mathrm{mmol})$, solvent $(2.0 \mathrm{~mL})$, under air for $6 \mathrm{~h}$.

a GC yields. ${ }^{b}$ Catalyst $\mathbf{1 b}(0.1 \mathrm{~mol} \%) \cdot{ }^{\mathrm{c}}$ Catalyst $\mathbf{2 a}(0.1 \mathrm{~mol} \%) .{ }^{\mathrm{d}}$ Catalyst $2 \mathbf{b}(0.1 \mathrm{~mol} \%)$.

Table 2

Heck cross-coupling reactions of aryl bromides with styrene.

\begin{tabular}{|c|c|c|c|c|c|c|c|c|c|}
\hline Entry & ArX & Product & $\begin{array}{c}\text { Yield }^{a} \\
(\%)\end{array}$ & $\begin{array}{c}\text { Yield b }^{\mathrm{b}} \\
(\%)\end{array}$ & Entry & $\operatorname{ArX}$ & Product & $\begin{array}{c}\text { Yield }^{a} \\
(\%)\end{array}$ & $\begin{array}{c}\text { Yield }^{\mathrm{b}} \\
(\%)\end{array}$ \\
\hline 1 & $\mathrm{C}_{6} \mathrm{H}_{5} \mathrm{Br}$ & & $>99$ & 95 & 11 & $p$-CHO- $\mathrm{C}_{6} \mathrm{H}_{4} \mathrm{Br}$ & & $>99$ & 96 \\
\hline 2 & $m-\mathrm{NH}_{2}-\mathrm{C}_{6} \mathrm{H}_{4} \mathrm{Br}$ & & 1.0 & & 12 & $o-\mathrm{CN}-\mathrm{C}_{6} \mathrm{H}_{4} \mathrm{Br}$ & & $>99$ & \\
\hline 3 & $p-\mathrm{NH}_{2}-\mathrm{C}_{6} \mathrm{H}_{4} \mathrm{Br}$ & & 3.0 & & 13 & $m-\mathrm{CN}-\mathrm{C}_{6} \mathrm{H}_{4} \mathrm{Br}$ & & $>99$ & \\
\hline 4 & $o-\mathrm{OCH}_{3}-\mathrm{C}_{6} \mathrm{H}_{4} \mathrm{Br}$ & & 4.0 & & 14 & $p$-CN- $\mathrm{C}_{6} \mathrm{H}_{4} \mathrm{Br}$ & & $>99$ & \\
\hline 5 & $p-\mathrm{OCH}_{3}-\mathrm{C}_{6} \mathrm{H}_{4} \mathrm{Br}$ & & 48 & & 15 & $p-\mathrm{COOH}-\mathrm{C}_{6} \mathrm{H}_{4} \mathrm{Br}$ & & 62 & \\
\hline 6 & $o-\mathrm{CH}_{3}-\mathrm{C}_{6} \mathrm{H}_{4} \mathrm{Br}$ & & 31 & & 16 & $p-\mathrm{CF}_{3}-\mathrm{C}_{6} \mathrm{H}_{4} \mathrm{Br}$ & & $>99$ & \\
\hline 7 & $p-\mathrm{CH}_{3}-\mathrm{C}_{6} \mathrm{H}_{4} \mathrm{Br}$ & & 54 & & 17 & $p-\mathrm{NO}_{2}-\mathrm{C}_{6} \mathrm{H}_{4} \mathrm{Br}$ & & $>99$ & \\
\hline 8 & $p-\mathrm{COOCH}_{3}-\mathrm{C}_{6} \mathrm{H}_{4} \mathrm{Br}$ & & 78 & 72 & 18 & 3-Br-pyridine & & 99 & \\
\hline 9 & $o-\mathrm{CHO}-\mathrm{C}_{6} \mathrm{H}_{4} \mathrm{Br}$ & & $>99$ & 95 & 19 & 3-Br-thiophene & & 71 & \\
\hline 10 & $m-\mathrm{CHO}-\mathrm{C}_{6} \mathrm{H}_{4} \mathrm{Br}$ & & $>99$ & 94 & 20 & $\begin{array}{c}\text { Bro- } \\
\text { mo-naphthalene }\end{array}$ & & 73 & \\
\hline
\end{tabular}

Reaction conditions: aryl bromides $(0.5 \mathrm{mmol})$, styrene $(2.0 \mathrm{mmol})$, catalyst 1a $(0.1 \mathrm{~mol} \%), \mathrm{NaOAc}(0.5 \mathrm{mmol}), n$-Bu $\mathrm{NBr}(0.5 \mathrm{mmol}), \mathrm{DMF}(2.0 \mathrm{~mL})$, at $130^{\circ} \mathrm{C}$ under air for $6 \mathrm{~h}$.

a $\mathrm{GC}$ yields. ${ }^{\mathrm{b}}$ Isolated yields. 
and 3-bromopyridine were used as substrates, the cross-coupling products were obtained in modest to excellent yields (entries 18-20). The results showed that complex 1a was an efficient catalyst for Heck cross-coupling reactions of aryl bromides in organic solvents.

\subsection{Heck coupling reactions in water}

The coupling reaction of 4-bromobenzene with styrene, catalyzed by palladacycle 1a, was selected as a model reaction. As shown in Table 3, when NaOAc was used as the base in water, the yield was excellent (entry 8). The relative activities of several palladacycles for the same model reaction, using $\mathrm{NaOAc}$ and water, were then studied, and we found that 1a exhibited the highest activity (entries 8-11).

We further investigated the Heck coupling reactions between a range of aryl bromides and styrene in water; the results are shown in Table 4. The reactions of electron-deficient aryl bromides with para substituents such as $-\mathrm{CN},-\mathrm{CHO},-\mathrm{NO}_{2}$, and $-\mathrm{CF}_{3}$ gave the coupling products in modest to high yields
Table 3

Investigation of Heck cross-coupling reaction conditions in water.

\begin{tabular}{|c|c|c|c|c|c|c|}
\hline Entry & Base & Solvent & $T\left({ }^{\circ} \mathrm{C}\right)$ & Yield a $\left.^{2} \%\right)$ & TON & TOF $\left(\mathrm{h}^{-1}\right)$ \\
\hline 1 & $\mathrm{Na}_{2} \mathrm{CO}_{3}$ & $\mathrm{H}_{2} \mathrm{O}$ & 100 & 71 & & \\
\hline 2 & $\mathrm{~K}_{3} \mathrm{PO}_{4}$ & $\mathrm{H}_{2} \mathrm{O}$ & 100 & 92 & & \\
\hline 3 & KOH & $\mathrm{H}_{2} \mathrm{O}$ & 100 & 88 & & \\
\hline 4 & $\mathrm{~K}_{3} \mathrm{PO}_{4} \cdot 3 \mathrm{H}_{2} \mathrm{O}$ & $\mathrm{H}_{2} \mathrm{O}$ & 100 & 94 & & \\
\hline 5 & $\left(\mathrm{CH}_{3}\right)_{3} \mathrm{ONa}$ & $\mathrm{H}_{2} \mathrm{O}$ & 100 & 88 & & \\
\hline 6 & $\mathrm{~K}_{2} \mathrm{CO}_{3}$ & $\mathrm{H}_{2} \mathrm{O}$ & 100 & 66 & & \\
\hline 7 & $\mathrm{NaHCO}_{3}$ & $\mathrm{H}_{2} \mathrm{O}$ & 100 & 67 & & \\
\hline 8 & $\mathrm{NaOAc}$ & $\mathrm{H}_{2} \mathrm{O}$ & 100 & $>99$ & 990 & 165 \\
\hline $9^{b}$ & $\mathrm{NaOAc}$ & $\mathrm{H}_{2} \mathrm{O}$ & 100 & 91 & 910 & 152 \\
\hline $10^{c}$ & $\mathrm{NaOAc}$ & $\mathrm{H}_{2} \mathrm{O}$ & 100 & 98 & 980 & 163 \\
\hline $11^{\mathrm{d}}$ & $\mathrm{NaOAc}$ & $\mathrm{H}_{2} \mathrm{O}$ & 100 & 93 & 930 & 155 \\
\hline 12 & $\mathrm{NaOAc}$ & $\mathrm{H}_{2} \mathrm{O}$ & 100 & 83 & & \\
\hline 13 & $\mathrm{NaOAc}$ & $\mathrm{H}_{2} \mathrm{O}$ & 90 & 90 & & \\
\hline
\end{tabular}

Reaction conditions: $\mathrm{PhBr}(0.5 \mathrm{mmol})$, styrene $(2 \mathrm{mmol})$, catalyst 1a (0.1 mol\%), base (0.5 mmol), $n$ - $\mathrm{Bu}_{4} \mathrm{NBr}(0.5 \mathrm{mmol}), \mathrm{H}_{2} \mathrm{O}(2.0 \mathrm{~mL})$, under air for 6 h. a GC yields. ${ }^{b}$ Catalyst $1 \mathbf{b}\left(0.1\right.$ mol\%). ${ }^{\mathrm{c}}$ Catalyst $2 \mathrm{2a}(0.1$ mol\%). ${ }^{\mathrm{d}}$ Catalyst $\mathbf{2 b}(0.1 \mathrm{~mol} \%)$.

\section{Table 4}

Heck cross-coupling reactions of aryl bromides with styrene in water.

\begin{tabular}{|c|c|c|c|c|c|c|c|c|c|}
\hline Entry $^{a}$ & $\operatorname{ArX}$ & Product & $\begin{array}{c}\text { Yield a }^{\text {(\%) }} \\
\end{array}$ & $\begin{array}{c}\text { Yield b } \\
(\%)\end{array}$ & Entry ${ }^{a}$ & ArX & Product & $\begin{array}{c}\text { Yield a } \\
(\%)\end{array}$ & $\begin{array}{c}\text { Yield b } \\
(\%)\end{array}$ \\
\hline 1 & $\mathrm{C}_{6} \mathrm{H}_{5} \mathrm{Br}$ & & $>99$ & 98 & 14 & $p-\mathrm{CF}_{3}-\mathrm{C}_{6} \mathrm{H}_{4} \mathrm{Br}$ & & 91 & 86 \\
\hline 2 & $o-\mathrm{CH}_{3}-\mathrm{C}_{6} \mathrm{H}_{4} \mathrm{Br}$ & & 5.0 & & 15 & $p-\mathrm{NO}_{2}-\mathrm{C}_{6} \mathrm{H}_{4} \mathrm{Br}$ & & 96 & 92 \\
\hline 3 & $p-\mathrm{CH}_{3}-\mathrm{C}_{6} \mathrm{H}_{4} \mathrm{Br}$ & & 10 & & 16 & 3-Br-pyridine & & 98 & 92 \\
\hline 4 & $m-\mathrm{NH}_{2}-\mathrm{C}_{6} \mathrm{H}_{4} \mathrm{Br}$ & & 32 & & 17 & 3-Br-furan & & 76 & \\
\hline 5 & $p-\mathrm{NH}_{2}-\mathrm{C}_{6} \mathrm{H}_{4} \mathrm{Br}$ & & 83 & 80 & 18 & 3-Br-thiophene & & 76 & \\
\hline 6 & $o-\mathrm{OCH}_{3}-\mathrm{C}_{6} \mathrm{H}_{4} \mathrm{Br}$ & & 5.0 & & 19 & $\begin{array}{c}\text { Bromo- } \\
\text { naphthalene }\end{array}$ & & 16 & \\
\hline 7 & $p-\mathrm{OCH}_{3}-\mathrm{C}_{6} \mathrm{H}_{4} \mathrm{Br}$ & & 7.0 & & 20 & $p-\mathrm{CH}_{3} \mathrm{CO}-\mathrm{C}_{6} \mathrm{H}_{4} \mathrm{Cl}$ & & 58 & \\
\hline 8 & $o-\mathrm{CHO}-\mathrm{C}_{6} \mathrm{H}_{4} \mathrm{Br}$ & & 34 & & 21 & $o-\mathrm{NO}_{2}-\mathrm{C}_{6} \mathrm{H}_{4} \mathrm{Cl}$ & & 28 & \\
\hline 9 & $m-\mathrm{CHO}-\mathrm{C}_{6} \mathrm{H}_{4} \mathrm{Br}$ & & 58 & & 22 & $\begin{array}{l}\text { 1-chloro-2,4- } \\
\text { dinitrobenzene }\end{array}$ & & 42 & \\
\hline 10 & $p$-CHO- $\mathrm{C}_{6} \mathrm{H}_{4} \mathrm{Br}$ & & 96 & 92 & 23 & $o-\mathrm{CH}_{3} \mathrm{O}-\mathrm{C}_{6} \mathrm{H}_{4} \mathrm{Cl}$ & & 7 & \\
\hline 11 & $o-\mathrm{CN}-\mathrm{C}_{6} \mathrm{H}_{4} \mathrm{Br}$ & & 51 & & 24 & $m-\mathrm{CH}_{3}-\mathrm{C}_{6} \mathrm{H}_{4} \mathrm{Cl}$ & & 23 & \\
\hline 12 & $m-\mathrm{CN}-\mathrm{C}_{6} \mathrm{H}_{4} \mathrm{Br}$ & & 33 & & 25 & $p-\mathrm{CH}_{3} \mathrm{O}-\mathrm{C}_{6} \mathrm{H}_{4} \mathrm{Cl}$ & & 80 & \\
\hline 13 & $p-\mathrm{CN}-\mathrm{C}_{6} \mathrm{H}_{4} \mathrm{Br}$ & & $>99$ & 94 & & & & & \\
\hline
\end{tabular}

Reaction conditions: aryl bromides (0.5 mmol), styrene (2.0 mmol), catalyst 1a (0.1 mol\%), NaOAc (0.5 mmol), $n$-Bu4 $\mathrm{NBr}(0.5 \mathrm{mmol}), \mathrm{DMF}(2.0 \mathrm{~mL})$, at $130{ }^{\circ} \mathrm{C}$ under air for $6 \mathrm{~h} .{ }^{a} \mathrm{GC}$ yields. ${ }^{\mathrm{b}}$ Isolated yields. 
(entries 1, 10, and 13-15), and those with ortho or meta substituents gave lower yields (entries 8, 9, 11, and 12). Aryl bromides with electron-rich groups such as $-\mathrm{OMe},-\mathrm{NH}_{2}$, and $-\mathrm{CH}_{3}$ exhibited lower reactivities under the same reaction condition (entries 2-4, 6, and 7), but 4-bromoaniline gave the coupling product in $83 \%$ yield (entry 5). When heteroaryl bromides such as 3-bromofuran and 3-bromopyridine were used as substrates, the coupling products were obtained in modest to excellent yields, but 1-bromonaphthalene was found to be a poor coupling partner under the same reaction conditions, giving only $16 \%$ yield (entries 16-19). Finally, the reactions of aryl chlorides with styrene in water were also investigated. Aryl chlorides gave the corresponding coupling products in low yields (entries 20-24). However, 1-chloro-4-methoxybenzene reacted with styrene to give the coupling product in $80 \%$ yield (entry 25).

\subsection{Suzuki cross-coupling reactions in organic solvents}

Suzuki cross-coupling reactions of 4-bromotoluene and phenylboronic acid were carried out using various bases and solvents in the presence of $0.1 \mathrm{~mol} \%$ of $1 \mathrm{a}$ without the protection of an inert gas; the results are shown in Table 5. As can be seen from Table 5, the reaction proceeded smoothly in toluene, and among the tested bases, $\mathrm{KOH}$ was found to be the most effective (entry 2). The relative activities of several palladacycles under the same reaction conditions were investigated, using $\mathrm{KOH}$ as the base and toluene as the solvent (entries 2-5). The palladacycles all exhibited high catalytic activities for Suzuki coupling reactions under the optimized reaction conditions.

Catalyst 1a was used to catalyze the Suzuki coupling reactions between aryl bromides and phenylboronic acid in toluene; the results are shown in Table 6. Electron-deficient or

Table 5

Investigation of Suzuki cross-coupling reaction conditions.

\begin{tabular}{|c|c|c|c|c|c|c|}
\hline Entry & Base & Solvent & $\begin{array}{c}T \\
\left({ }^{\circ} \mathrm{C}\right)\end{array}$ & $\begin{array}{c}\text { Yield a } \\
(\%) \\
\end{array}$ & TON & $\begin{array}{l}\text { TOF } \\
\left(h^{-1}\right)\end{array}$ \\
\hline 1 & $\mathrm{~K}_{2} \mathrm{CO}_{3}$ & toluene & 100 & 47 & & \\
\hline 2 & $\mathrm{KOH}$ & toluene & 100 & 96 & 960 & 480 \\
\hline $3^{b}$ & $\mathrm{KOH}$ & toluene & 100 & 93 & 930 & 465 \\
\hline $4^{c}$ & $\mathrm{KOH}$ & toluene & 100 & 91 & 910 & 455 \\
\hline $5^{d}$ & $\mathrm{KOH}$ & toluene & 100 & 94 & 940 & 470 \\
\hline 6 & $\mathrm{~K}_{3} \mathrm{PO}_{4} \cdot 3 \mathrm{H}_{2} \mathrm{O}$ & toluene & 100 & 81 & & \\
\hline 7 & $\mathrm{~K}_{3} \mathrm{PO}_{4}$ & toluene & 100 & 88 & & \\
\hline 8 & $\mathrm{~K}_{3} \mathrm{PO}_{4} \cdot 7 \mathrm{H}_{2} \mathrm{O}$ & toluene & 100 & 92 & & \\
\hline 9 & $\mathrm{Na}_{2} \mathrm{CO}_{3}$ & toluene & 100 & 0.2 & & \\
\hline 10 & KOH & dioxane & 100 & 74 & & \\
\hline 11 & $\mathrm{KOH}$ & DMF & 120 & 17 & & \\
\hline 12 & $\mathrm{KOH}$ & $\mathrm{THF}$ & 70 & 15 & & \\
\hline 13 & $\mathrm{KOH}$ & $\mathrm{H}_{2} \mathrm{O}$ & 90 & 77 & & \\
\hline 14 & $\mathrm{KOH}$ & methanol & 70 & 90 & & \\
\hline
\end{tabular}

Reaction conditions: 4-bromotoluene ( $0.5 \mathrm{mmol}), \mathrm{PhB}(\mathrm{OH})_{2}(0.6 \mathrm{mmol})$, base $(1.0 \mathrm{mmol})$, solvent $(2.0 \mathrm{~mL})$, catalyst $1 \mathrm{a}(0.1 \mathrm{~mol} \%)$, under air for $2 \mathrm{~h}$.

a GC yields. ${ }^{\mathrm{b}}$ Catalyst $\mathbf{1 b}(0.1 \mathrm{~mol} \%) .{ }^{\mathrm{c}}$ Catalyst $\mathbf{2 a}(0.1 \mathrm{~mol} \%) .{ }^{\mathrm{d}}$ Catalyst 2 b $(0.1 \mathrm{~mol} \%)$.
Table 6

Suzuki coupling reactions of aryl halides with phenylboronic acid.

\begin{tabular}{|c|c|c|c|c|}
\hline Entry & ArX & Product & Yield a (\%) & Yield ${ }^{\mathrm{b}}(\%)$ \\
\hline 1 & $o-\mathrm{CH}_{3}-\mathrm{C}_{6} \mathrm{H}_{4} \mathrm{Br}$ & & 44 & \\
\hline 2 & $p-\mathrm{CH}_{3}-\mathrm{C}_{6} \mathrm{H}_{4} \mathrm{Br}$ & & 96 & 94 \\
\hline 3 & $m-\mathrm{NH}_{2}-\mathrm{C}_{6} \mathrm{H}_{4} \mathrm{Br}$ & & 41 & \\
\hline 4 & $p-\mathrm{NH}_{2}-\mathrm{C}_{6} \mathrm{H}_{4} \mathrm{Br}$ & & 45 & \\
\hline 5 & $p-\mathrm{CF}_{3}-\mathrm{C}_{6} \mathrm{H}_{4} \mathrm{Br}$ & & $>99$ & 93 \\
\hline 6 & $p-\mathrm{NO}_{2}-\mathrm{C}_{6} \mathrm{H}_{4} \mathrm{Br}$ & & 92 & 90 \\
\hline 7 & $o-\mathrm{CN}-\mathrm{C}_{6} \mathrm{H}_{4} \mathrm{Br}$ & & 80 & \\
\hline 8 & $m-\mathrm{CN}-\mathrm{C}_{6} \mathrm{H}_{4} \mathrm{Br}$ & & 56 & \\
\hline 9 & $p-\mathrm{CN}-\mathrm{C}_{6} \mathrm{H}_{4} \mathrm{Br}$ & & 92 & 88 \\
\hline 10 & $p-\mathrm{COCH}_{3}-\mathrm{C}_{6} \mathrm{H}_{4} \mathrm{Br}$ & & 95 & \\
\hline 11 & 3-Br-pyridine & & 99 & \\
\hline 12 & 3-Br-thiophene & & $>99$ & 95 \\
\hline 13 & $\begin{array}{c}\text { Bromo- } \\
\text { naphthalene }\end{array}$ & & 58 & \\
\hline
\end{tabular}

Reaction conditions: aryl bromide $(0.5 \mathrm{mmol}), \mathrm{PhB}(\mathrm{OH})_{2}(0.6 \mathrm{mmol})$, $\mathrm{KOH}(1.0 \mathrm{mmol})$, toluene $(2.0 \mathrm{~mL})$, catalyst $1 \mathrm{a}(0.1 \mathrm{~mol} \%)$, at $100{ }^{\circ} \mathrm{C}$ under air for $2 \mathrm{~h}$. ${ }^{\mathrm{a}} \mathrm{GC}$ yields. ${ }^{\mathrm{b}}$ Isolated yields.

electron-rich aryl bromides containing groups such as $-\mathrm{CN}$, $-\mathrm{CHO},-\mathrm{NO}_{2},-\mathrm{CF}_{3}$, and $-\mathrm{CH}_{3}$ at the para position gave the coupling products in good yields (entries 2, 5, 6, 9, and 10), and those with ortho or meta substituents gave the coupling products in moderate to good yields (entries 1, 3, 7, and 8). 3-Bromofuran and 3-bromopyridine gave the coupling products in excellent yields, but 1-bromonaphthalene gave a yield of $58 \%$ (entries 11-13).

\subsection{Suzuki coupling reactions in pure water}

The catalytic properties of the palladacycles in Suzuki coupling reactions in water were investigated. The reaction conditions were screened using the coupling reaction of bromobenzene with phenylboronic acid, using $0.1 \mathrm{~mol} \%$ of $\mathbf{1 a}$ as a model reaction. As shown in Table $7, \mathrm{~K}_{3} \mathrm{PO}_{4} \cdot 3 \mathrm{H}_{2} \mathrm{O}, \mathrm{NaHCO}_{3}$, and $\mathrm{K}_{3} \mathrm{PO}_{4} \cdot 7 \mathrm{H}_{2} \mathrm{O}$ were found to be effective bases, giving high yields in water at $100{ }^{\circ} \mathrm{C}$ (entries 4,7 , and 9). When the reaction temperature was reduced from $100{ }^{\circ} \mathrm{C}$ to 90 and $80{ }^{\circ} \mathrm{C}$, isolated yields of $97 \%$ and $89 \%$, respectively, were obtained (entries 10 and 11). The relative activities of several palladacycles were investigated under the same reaction conditions using $\mathrm{K}_{3} \mathrm{PO}_{4} \cdot 3 \mathrm{H}_{2} \mathrm{O}$ (entries 10-13). The palladacycles all exhibited high catalytic activities for Suzuki coupling reactions under the 
Table 7

Investigation of Suzuki cross-coupling reaction conditions in water.

\begin{tabular}{|c|c|c|c|c|c|c|}
\hline Entry & Base & Solvent & $T\left({ }^{\circ} \mathrm{C}\right)$ & Yield a (\%) & TON & TOF $\left(\mathrm{h}^{-1}\right)$ \\
\hline 1 & $\mathrm{Na}_{2} \mathrm{CO}_{3}$ & $\mathrm{H}_{2} \mathrm{O}$ & 100 & 82 & & \\
\hline 2 & $\mathrm{~K}_{3} \mathrm{PO}_{4}$ & $\mathrm{H}_{2} \mathrm{O}$ & 100 & 70 & & \\
\hline 3 & $\mathrm{KOH}$ & $\mathrm{H}_{2} \mathrm{O}$ & 100 & 89 & & \\
\hline 4 & $\mathrm{~K}_{3} \mathrm{PO}_{4} \cdot 3 \mathrm{H}_{2} \mathrm{O}$ & $\mathrm{H}_{2} \mathrm{O}$ & 100 & 97 & & \\
\hline 5 & $\mathrm{Et}_{3} \mathrm{~N}$ & $\mathrm{H}_{2} \mathrm{O}$ & 100 & 85 & & \\
\hline 6 & $\mathrm{~K}_{2} \mathrm{CO}_{3}$ & $\mathrm{H}_{2} \mathrm{O}$ & 100 & 45 & & \\
\hline 7 & $\mathrm{NaHCO}_{3}$ & $\mathrm{H}_{2} \mathrm{O}$ & 100 & 96 & & \\
\hline 8 & $\mathrm{NaOAc}$ & $\mathrm{H}_{2} \mathrm{O}$ & 100 & 67 & & \\
\hline 9 & $\mathrm{~K}_{3} \mathrm{PO}_{4} \cdot 7 \mathrm{H}_{2} \mathrm{O}$ & $\mathrm{H}_{2} \mathrm{O}$ & 100 & 96 & & \\
\hline 10 & $\mathrm{~K}_{3} \mathrm{PO}_{4} \cdot 3 \mathrm{H}_{2} \mathrm{O}$ & $\mathrm{H}_{2} \mathrm{O}$ & 90 & 97 & 970 & 485 \\
\hline $11^{\mathrm{b}}$ & $\mathrm{K}_{3} \mathrm{PO}_{4}: 3 \mathrm{H}_{2} \mathrm{O}$ & $\mathrm{H}_{2} \mathrm{O}$ & 90 & 92 & 920 & 460 \\
\hline $12^{c}$ & $\mathrm{~K}_{3} \mathrm{PO}_{4} \cdot 3 \mathrm{H}_{2} \mathrm{O}$ & $\mathrm{H}_{2} \mathrm{O}$ & 90 & 94 & 940 & 470 \\
\hline $13^{\mathrm{d}}$ & $\mathrm{K}_{3} \mathrm{PO}_{4} \cdot 3 \mathrm{H}_{2} \mathrm{O}$ & $\mathrm{H}_{2} \mathrm{O}$ & 90 & 94 & 940 & 470 \\
\hline 14 & $\mathrm{~K}_{3} \mathrm{PO}_{4} \cdot 3 \mathrm{H}_{2} \mathrm{O}$ & $\mathrm{H}_{2} \mathrm{O}$ & 80 & 89 & & \\
\hline
\end{tabular}

Reaction condition: bromobenzene $(0.5 \mathrm{mmol}), \mathrm{PhB}(\mathrm{OH})_{2}(0.6 \mathrm{mmol})$, base (1.0 mmol), $\mathrm{H}_{2} \mathrm{O}(2.0 \mathrm{~mL})$, catalyst $1 \mathrm{a}(0.1 \mathrm{~mol} \%)$, under air for $2 \mathrm{~h}$. ${ }^{a}$ GC yields. ${ }^{b}$ Catalyst $\mathbf{1 b}(0.1 \mathrm{~mol} \%) .{ }^{\mathrm{c}}$ Catalyst $\mathbf{2 a}(0.1 \mathrm{~mol} \%) .{ }^{\mathrm{d}}$ Catalyst $2 \mathbf{b}(0.1 \mathrm{~mol} \%)$.

optimized reaction conditions.

We next examined the scope and limitations of the protocol using aryl bromides and phenylboronic acid in the presence of $0.1 \mathrm{~mol} \% \mathrm{1a}$ at $90{ }^{\circ} \mathrm{C}$ with $\mathrm{K}_{3} \mathrm{PO}_{4} \cdot 3 \mathrm{H}_{2} \mathrm{O}$ as the base. The obtained results are summarized in Table 8. Aryl bromides with electron-donating and electron-withdrawing para substituents all worked well, providing the target products in good to excellent yields after $2 \mathrm{~h}$ (entries 2, 4, 6, 8, 12, and 13). The coupling reactions also proceeded smoothly with 3-bromopyridine, 3-bromothiophene, and 1-bromonaphthalene under the same reaction conditions to afford the coupling products in modest
Table 9

Suzuki coupling reactions of aryl chlorides and iodides with phenylboronic acid in water.

Entry

Reaction conditions: aryl chlorides or iodides $(0.5 \mathrm{mmol}), \mathrm{PhB}(\mathrm{OH})_{2}$ (0.6 mmol), $\mathrm{K}_{3} \mathrm{PO}_{4}: 3 \mathrm{H}_{2} \mathrm{O}(1.0 \mathrm{mmol}), \mathrm{H}_{2} \mathrm{O}(2.0 \mathrm{~mL})$, catalyst $\mathbf{1 a}(0.1$

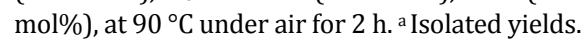

to good yields (entries 14-16). However, sterically hindered aryl bromides containing ortho or meta substituents gave the coupled product in slightly lower yields (entries 1, 3, 5, and 7). Interestingly, 2-bromobenzonitrile, 3-bromobenzonitrile, and 4-bromobenzonitrile provided the coupled product in $96 \%$, $76 \%$, and $66 \%$ yields, respectively (entries 9-11).

Finally, the reactions of aryl chlorides or iodides with phenylboronic acid in water were also tested. The results are summarized in Table 9. Aryl chlorides gave the corresponding coupling products in low yields (entries 1-4). Iodobenzene reacted with phenylboronic acid to give the coupling product in

Table 8

Suzuki coupling reactions of aryl bromides with phenylboronic acid in water.

\begin{tabular}{|c|c|c|c|c|c|c|c|c|c|}
\hline Entry & ArX & Product & $\begin{array}{c}\text { Yield }^{a} \\
(\%)\end{array}$ & $\begin{array}{c}\text { Yield b }^{\text {b }} \\
\text { (\%) }\end{array}$ & Entry & ArX & Product & $\begin{array}{c}\text { Yield }^{\text {a }} \\
(\%)\end{array}$ & $\begin{array}{c}\text { Yield }^{\mathrm{b}} \\
(\%)\end{array}$ \\
\hline 1 & $o-\mathrm{CH}_{3}-\mathrm{C}_{6} \mathrm{H}_{4} \mathrm{Br}$ & & 67 & & 9 & $o-\mathrm{CN}-\mathrm{C}_{6} \mathrm{H}_{4} \mathrm{Br}$ & & 96 & \\
\hline 2 & $p-\mathrm{CH}_{3}-\mathrm{C}_{6} \mathrm{H}_{4} \mathrm{Br}$ & & 97 & 94 & 10 & $m-\mathrm{CN}-\mathrm{C}_{6} \mathrm{H}_{4} \mathrm{Br}$ & & 76 & \\
\hline 3 & $m-\mathrm{NH}_{2}-\mathrm{C}_{6} \mathrm{H}_{4} \mathrm{Br}$ & & 60 & & 11 & $p-\mathrm{CN}-\mathrm{C}_{6} \mathrm{H}_{4} \mathrm{Br}$ & & 86 & 85 \\
\hline 4 & $p-\mathrm{NH}_{2}-\mathrm{C}_{6} \mathrm{H}_{4} \mathrm{Br}$ & & 91 & & 12 & $p-\mathrm{CF}_{3}-\mathrm{C}_{6} \mathrm{H}_{4} \mathrm{Br}$ & & 99 & 95 \\
\hline 5 & $o-\mathrm{OCH}_{3}-\mathrm{C}_{6} \mathrm{H}_{4} \mathrm{Br}$ & & 47 & & 13 & $p-\mathrm{NO}_{2}-\mathrm{C}_{6} \mathrm{H}_{4} \mathrm{Br}$ & & 92 & \\
\hline 6 & $p-\mathrm{OCH}_{3}-\mathrm{C}_{6} \mathrm{H}_{4} \mathrm{Br}$ & & 71 & 68 & 14 & 3-Br-pyridine & & 71 & \\
\hline 7 & $o-\mathrm{CHO}-\mathrm{C}_{6} \mathrm{H}_{4} \mathrm{Br}$ & & 75 & & 15 & 3-Br-thiophene & & 85 & \\
\hline 8 & $p-\mathrm{CHO}-\mathrm{C}_{6} \mathrm{H}_{4} \mathrm{Br}$ & & 87 & 82 & 16 & Bromo-naphthalene & & 65 & \\
\hline
\end{tabular}


$79 \%$ yield, and 4-iodobenzoic acid was found to be a poor coupling partner (entries 5 and 6). This is probably because 4-iodobenzoic acid was highly reactive and failed to undergo cross-coupling in our catalytic system.

\section{Conclusions}

A series of cyclopalladated ferrocenylimine dimers and monomers with ester groups were designed and prepared. We also developed a convenient and efficient methodology for Suzuki and Heck coupling reactions of aryl bromides catalyzed by cyclopalladated ferrocenylimines with ester groups in organic solvents or water under ambient conditions with low catalyst loadings of $0.1 \mathrm{~mol} \%$. Suzuki coupling reactions in water at 90 ${ }^{\circ} \mathrm{C}$ in the absence of additives are significant in terms of both economics and the environment.

\section{References}

[1] Suzuki A. J Organomet Chem, 1999, 576: 147

[2] Wolfe J P, Singer R A, Yang B H, Buchwald S L. J Am Chem Soc, 1999, 121: 9550

[3] Miyaura N. Top Curr Chem, 2002, 219: 11

[4] Hassan J, Sevignon M, Gozzi C, Schulz E, Lemaire M. Chem Rev, 2002, 102: 1359

[5] Stille J K. Angew Chem Int Ed, 1986, 25: 508

[6] Beller M, Fischer H, Herrmannl W A, Öfele K, Brossmer1 C, Reisinger C P, Priermeier T. Angew Chem Int Ed, 1995, 34: 1848

[7] Sheldon R A, van Bekkum H. Fine Chemicals through Heterogeneous Catalysis. Weinheim: Wiley-VCH, 2001

[8] Nicolaou K C, Boddy C N C, Brase S, Winssinger N. Angew Chem Int Ed, 1999, 38: 2096

[9] Baudoin O, Cesario M, Guenard D, Gueritte F.J Org Chem, 2002, 67: 1199

[10] Beletskaya I P, Cheprakov A V C. Chem Rev, 2000, 100: 3009

[11] Yao Q W, Kinney E P, Yang Z.J Org Chem, 2003, 68: 7528

[12] Zhao N, Wang F, Zhou M L, Li T S, Liu H, Xu W J, Wu Y J. Chin J Catal (赵娜, 王飞, 周梅玲, 李铁生, 刘辉, 许文俭, 吴养洁. 催化学报), 2013, 34: 1583

[13] Ge Y C, Cheng Y J, Fu H Y, Zheng X L, Li R X, Chen H, Li X J. Chin J Catal (葛轶苓, 程雨晶, 付海燕, 郑学丽, 李瑞祥, 陈华, 李贤均. 催化 学报), 2013, 34: 1667

[14] Kovala-Demertzi D, Yadav P N, Demertzis M A, Jasiski J P,
Andreadaki F J, Kostas I D. Tetrahedron Lett, 2004, 45: 2923

[15] Tundo P, Anastas P, Black D S, Breen J, Collins T, Memoli S, Miyamoto J, Polyakoff M, Tumas W. Pure Appl Chem, 2000, 72: 1207

[16] Liu L F, Zhang Y H, Wang Y G. J Org Chem, 2005, 70: 6122

[17] Liu L F, Zhang Y H, Xin B W. J Org Chem, 2006, 71: 3994

[18] Li H, Wu Y J. Appl Organomet Chem, 2008, 22: 233

[19] Zhou J, Guo X M, Tu C Z, Li X Y, Sun H J. J Organomet Chem, 2009, 694: 697

[20] Saha D, Chattopadhyay K, Ranu B C. Tetrahedron Lett, 2009, 50 : 1003

[21] Basu B, Biswas K, Kundu S, Ghosh S. Green Chem, 2010, 12: 1734

[22] Veerakumar P, Velayudham M, Lu K L, Rajagopal S. Appl Catal A, 2013, 455: 247

[23] Wójcik K, Goux-Henry C, Andrioletti B, Pietrusiewicz K M, Framery E. Tetrahedron Lett, 2012, 53: 5602

[24] Konovets A, Penciu A, Framery E, Percina N, Goux-Henry C, Sinou D. Tetrahedron Lett, 2005, 46: 3205

[25] Shang Y J, Wu J W, Fan C L, Hu J S, Lu B Y. J Organomet Chem, 2008, 693: 2963

[26] Mu B, Li J Y, Han Z X, Wu Y J. J Organomet Chem, 2012, 700: 117

[27] Schönfelder D, Nuyken 0, Weberskirch R. J Organomet Chem, 2005, 690: 4648

[28] Susanto W, Chu C Y, Ang W J, Chou T C, Lo L C, Lam Y.J Org Chem, 2012, 77: 2729

[29] Zhang J L, Yang F, Ren G R, Mak T C W, Song M P, Wu Y J. Ultrason Sonochem, 2008, 15: 115

[30] Marziale A N, Jantke D, Faul S H, Reiner T, Herdtweck E, Eppinger J. Green Chem, 2011, 13: 169

[31] Yang J H, Wang D F, Liu W D, Zhang X, Bian F L, Yu W. Green Chem, 2013, 15: 3429

[32] El Bakouri O, Fernández M, Brun S, Pla-Quintana A, Roglans A. Tetrahedron, 2013, 69: 9761

[33] Mu B, Li T S, Li J Y, Wu Y J. J Organomet Chem, 2008, 693: 1243

[34] Mu B, Li T S, Fu Z H, Wu Y J. Catal Commun, 2009, 10: 1497

[35] Yu A J, Shen L, Cui X L, Peng D P, Wu Y J. Tetrahedron, 2012, 68: 2283

[36] Huang M M, Feng Y J, Wu Y J. Tetrahedron, 2012, 68: 376

[37] Zhang J L, Yang X Q, Cui X L, Wu Y J. Tetrahedron, 2011, 67: 8800

[38] Leng Y T, Yang F, Zhu W G, Zou D P, Wu Y J, Cai R R. Tetrahedron, 2011, 67: 6191

[39] Mu B, Li T S, Li C H, Liu P P, Shang W, Wu Y J. Tetrahedron, 2009, 65: 2599

[40] Yang F, Cui X L, Li Y N, Zhang J L, Ren G R, Wu Y J. Tetrahedron, 2007, 63: 1963

[41] Li J Y, Cui M J, Yu A J, Wu Y J. J Organomet Chem, 2007, 692: 3732

\section{Graphical Abstract}

Chin. J. Catal., 2014, 35: 1059-1067 doi: 10.1016/S1872-2067(14)60045-0

\section{Cyclopalladated ferrocenylimines with ester group for Heck and Suzuki coupling reactions}

Jiong Wang, Bing Mu, Zhihua Fu, Li Wang, Tiesheng Li*, Yangjie Wu* Zhengzhou University; Zhengzhou Normal University

Cyclopalladated ferrocenylimines with ester groups were prepared and effectively catalyzed Heck and Suzuki coupling reactions of aryl or heteroaryl halides in water or organic solvents.

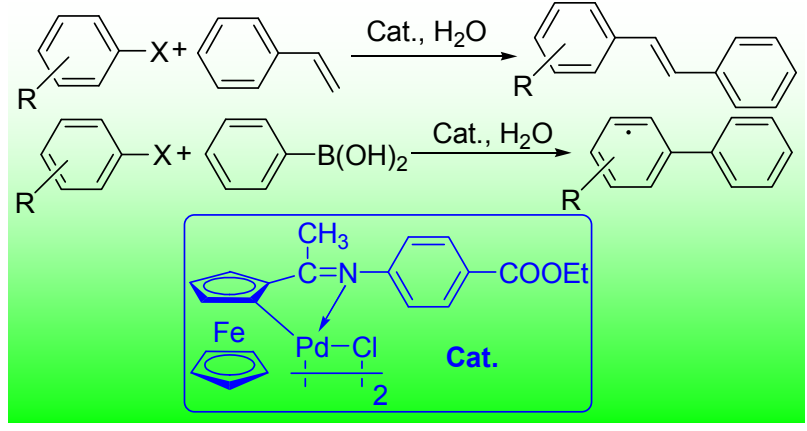




\title{
含有酯基二茂铁亚胺环钯化合物催化Suzuki和Heck反应
}

\author{
王 畑 ${ }^{\mathrm{a}}$, 穆 兵 ${ }^{\mathrm{b}}$, 付志华 ${ }^{\mathrm{a}}$, 汪 网 $^{\mathrm{a}}$, 李铁生 ${ }^{\mathrm{a},{ }^{*}}$, 吴养洁 ${ }^{\mathrm{a}, \#}$
}

a郑州大学化学与分子工程学院, 河南省化学生物与有机化学重点实验室, 郑州大学先进纳米信息材料实验室, 河南郑州450052 b 郑州师范学院化学系, 河南郑州 450044

摘要: 合成并表征了系列含有酯基二茂铁亚胺环钯化合物. 结果表明, 该系列环钯催化剂在纯水或有机溶剂中均能高效催化 Heck和Suzuki偶联反应.

关键词: Suzuki偶联反应; Heck偶联反应; 环钯二茂铁亚胺

收稿日期: 2013-10-10. 接受日期: 2014-01-22. 出版日期: 2014-07-20.

*通讯联系人. 电话/传真: (0371)67766667; 电子信箱: 1ts34@zzu.edu.cn

\#通讯联系人. 电话/传真: (0371)67766667; 电子信箱: wyj@zzu.edu.cn

基金来源: 国家自然科学基金(20973157, J1210060); 郑州大学创新研究基金(2013xjxm020).

本文的英文电子版由Elsevier出版社在ScienceDirect上出版(http://www.sciencedirect.com/science/journal/18722067). 